\title{
Optical properties of inverted opal photonic band gap crystals with stacking disorder
}

\author{
Z. L. Wang, ${ }^{1}$ C. T. Chan, ${ }^{2}$ W. Y. Zhang, ${ }^{1}$ Z. Chen, ${ }^{1}$ N. B. Ming, ${ }^{1}$ and P. Sheng ${ }^{2}$ \\ ${ }^{1}$ National Laboratory of Solid State Microstructures, Nanjing University, Nanjing 210093, China \\ ${ }^{2}$ Department of Physics, Hong Kong University of Science and Technology, Clear Water Bay, Kowloon, Hong Kong, China
}

(Received 27 November 2001; revised manuscript received 5 November 2002; published 31 January 2003)

\begin{abstract}
We present a detailed study of the effect of planar stacking disorder on optical properties of inverted opal photonic crystals. Systems with periodic stacking sequences are first studied that include face centered cubic, hexagonal close-packed, and doubly hexagonal close-packed photonic crystals. For the structures with periodic stacking order, we evaluate the band structure followed by calculation of transmission spectrum along the direction perpendicular to the hexagonal close-packing plane of the structures. Inverted opal photonic crystals with random stacking sequences are then studied by calculating average transmittance of the photonic crystal slabs over various random stacking configurations. The position and width of the lowest stop gap along the direction normal to the hexagonal close-packing plane is found to be invariant, regardless of the stacking sequence in the photonic crystal. We show how the propagation properties at higher frequencies are affected by the stacking configurations (both periodic and disordered stacking sequences), particularly those near the edges of the absolute band gap. The obtained results are directly relevant to transmission/reflection experiments on inverted opal photonic crystals with complete band gap.
\end{abstract}

DOI: 10.1103/PhysRevE.67.016612

PACS number(s): 42.70.Qs, 42.25.Bs, 82.70.Dd

\section{INTRODUCTION}

Materials whose refractive index is modulated with a period on the order of the desired wavelength of operation have attracted much attention over the last decade $[1,2]$. These structures, known as photonic crystals, give rise to strong coherent multiple scattering of electromagnetic (EM) waves within the material. As a result, a frequency region of spectrum (stop gap) opens up in which light cannot propagate. If the scattering is strong enough, the stop gaps in all directions will overlap, leading to the formation of a complete photonic band gap (CPBG) in which EM wave propagation is forbidden irrespective of propagation direction and photon polarization. Recently, considerable effort has been made in design, fabrication, and numerical simulation of artificial crystals because CPBG structures promise to be an important ingredient in the development of optical devices with novel properties [3].

In order to open a CPBG in the visible/near-infrared region of light spectrum, modulation period of refractive index must be less than $1 \mu \mathrm{m}$. In this regard, colloidal particles are believed to be the fast and cheap building blocks for photonic crystals owing to their submicrometer sizes and selforganization property. Monodispersed spherical microparticles can self-assemble into a three-dimensional (3D) closepacked array to create an opal that was shown to display photonic gap effect [4-6]. Although these synthesized 3D artificial structures exhibit only a stop gap, theoretical studies [7-9] have shown that a CPBG exists in the inverted structure. This system consists of a face centered cubic (FCC) crystalline of low dielectric spherical scatterers with refractive index $n_{s}$ in an interconnected high dielectric matrix with refractive index $n_{b}$. These predictions have triggered extensive experimental work to use opal as matrices and to fill the voids in it with materials of high refractive index to fabricate CPBG crystals in the visible and near IR range of light [1012]. In order to increase the contrast in refractive index, an "air-sphere" structure can be obtained by removing the dielectric spheres after the infiltration process $[13,14]$. These artificial structures (hereafter called inverted opal), of which the refractive index contrast $\delta=n_{b} / n_{s}$ is normally lower than 2.6, have shown a strong photonic effect [15-18]. The fabrication of an inverted opal with a CPBG in the near IR range by means of opal templating techniques has been recently reported $[19,20]$.

It is well known that synthetic opals have intrinsic disorder including stacking faults, lattice dislocations, and point defects. Vlasov et al. [21] first studied the influence of various types of defects, especially the stacking faults, on optical properties of synthetic opals by means of study of transmission, reflection, and diffraction along different crystallographic directions. Although the point defects can be much reduced by using colloidal particles with narrow size distribution, stacking faults are inevitable in the self-organization process. As an illustration of the stacking disorder, let $A$ denote a hexagonal close-packing layer; normally the growth direction of opals is perpendicular to this dense hexagonal plane. Now there are two possibilities that the next layer can be placed: positions $B$ and $C$. Thermodynamical studies have shown that the difference in free energy between these two stacking configurations is negligibly small [22]; the resulting structure may not be pure FCC with repeating $A B C$ stacking sequence along the growth direction $[17,23]$. It could be hexagonal close-packing structure (HCP) with $A B A B \ldots$ stacking sequence, or a double HCP structure (DHCP) with $A B C B A B C B \ldots$ stacking sequence containing periodic stacking faults as compared to the FCC structure. Furthermore, studies of concentrated colloidal dispersion show that the most common type of structures in a shear ordered colloidal crystal is close to a random stacking of close-packed planes [24,25] with a stacking sequence such as $A B C B A B A C A B A C \ldots$

Because an inverted opal is created by the direct templating of opal, the produced macroporous materials preserve all 
the disorder introduced in the original opals. Unless the photonic band gap is robust enough to survive these inevitable disorders, the potential for application of these inverted opal crystals would be compromised. Recent supercell simulation showed that the optical properties of an inverted opal are affected by geometrical nonuniformity. The CPBG could be destroyed by a moderate amount of disorder in the sizes and positions of the air spheres [26]. Yannopapas et al. [27] have very recently shown that the introduction of a certain amount of stacking faults in an inverted FCC opal is associated with some very interesting property such as the light localization due to disorder.

In this paper we investigate in detail the optical properties of an inverted opal photonic crystal with both periodic and random planar stacking sequences. For structures with ordered stacking sequences both band structure and transmission spectra are presented. The periodic stacking structures to be studied include inverted FCC, HCP, and DHCP opals, which contain three, two, and four planes of spheres in the stacking unit, respectively. For these periodic systems, we find gaps and nonoptically active bands in the band structures along the axis perpendicular to the hexagonal closepacking planes, which are further verified in evaluation of transmission spectra. We then discuss how the transmission property is affected by introducing random planar stacking in the photonic crystal slab. We found that the transmission spectra of random systems show strong dips at nearly the same frequencies as periodic systems do. In addition, large fluctuations in the transmittance are observed at the edges of the stop gaps at higher frequencies for random systems. We also show that the nonoptically active range of frequencies observed in the transmission spectra under normal incidence for periodic structures become coupled to the incident light by applying off-normal incidence or by breaking the symmetry along the stacking axis via the introduction of stacking disorder.

\section{DISCUSSION AND RESULTS}

The multiple scattering method is probably best suited to calculate the band structure of our system [28-30]. This method is accurate and highly efficient as the scattering matrix of each sphere can be handled analytically. The only requirement is that the spheres do not overlap. In order to evaluate the transmission/reflection spectrum of PBG materials with a finite thickness, we employ the on-shell method $[31,32]$, in which the transmission and reflection coefficients of a homogeneous plate or a multilayer of spherical particles of given periodicity parallel to the surface can be calculated.

The crystal slabs considered in our paper can be seen as a successive stacking of identical planes of close-packed air spheres embedded in a host medium of GaAs, which are shown in Fig. 1. The neighboring planes of spheres are separated at a distance $d=\sqrt{6} \alpha / 3$, where $\alpha$ is the nearest neighbor distance in the 2D plane of air spheres. The identical layers of air spheres are further assumed to be parallel to the surfaces of host medium. We restrict our considerations to the cases where the first and last layers of air spheres are situated at the same distance $d_{s}$ from the front and end sur-

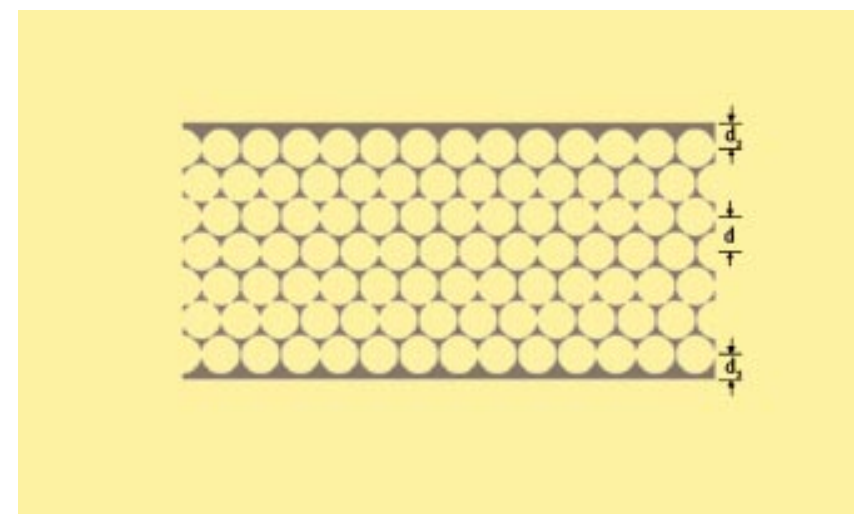

FIG. 1. Schematic representation of an inverted opal in the matrix of gallium arsenide. $d$ denotes the distance between neighboring layers of air spheres in the structure. The front and end surfaces of the host medium are assumed to be situated at the same distance $d_{s}$ from the first and last layers of air spheres, respectively.

face of the host matrix, respectively. We choose the axes such that the layers of air spheres lie in the $x-y$ plane. The $z$ direction, which is perpendicular to the planes of air spheres, can be referred to as [111] in FCC and [0001] in HCP and DHCP notations (see, e.g., Ref. [21]).

In the following discussion, we use a dimensionless frequency $\tilde{\omega}=\omega D / 2 \pi c$, where $D$ is the diameter of air sphere and $c$ the speed of light in vacuum and can be expressed as $D=2 \sqrt{2}(3 f / 16 \pi)^{1 / 3} \alpha$ with $f$ being the volume filling fraction of air spheres. The adoption of this unit will allow for a direct comparison of photonic properties among structures with different stacking sequences. In all the numerical simulations, we set the parameter $d_{s}=D / 2$ and assume an array of touching air spheres (filling ratio $f=0.74$ ) in GaAs (refractive index $n_{b}=3.6$ ). Furthermore, we are mainly concerned with the transmission properties under normal incidence, which are measured in most experiments [15-17,19,20], though some calculations for the case of off-normal incidence for the FCC structures are also presented. In our band structure evaluations, excellent convergence is achieved by including spherical waves with angular momentum up to $l_{\max }=7$ in the local expansion of EM waves. The transmission calculation was performed using $l_{\max }=7$ in the angular momentum expansions and 37 2D reciprocal lattice vectors in the plane-wave expansions. This gives a relative accuracy of $10^{-5}$ for the transmittance of an individual slab [27].

\section{A. Inverted opal with FCC structure}

In Fig. 2 we present the band structure of an inverted opal photonic crystal with FCC geometry. It is known that this structure exhibits a CPBG when the refractive index of the host medium $n_{b}>2.8[8,9]$. As can be seen here, the FCC structure possesses a CPBG centered at $\tilde{\omega}=0.542$ with a relative size $\Delta \tilde{\omega} / \tilde{\omega}_{0}=5.7 \%$ between the eighth and ninth bands. Along the $\Gamma L$ symmetry line which corresponds to the [111] stacking axis in FCC, the structure develops three gaps. Counting from low to high frequencies, the first gap is at $\tilde{\omega}$ $\approx 0.288-0.365$ between the second and third bands, the sec- 


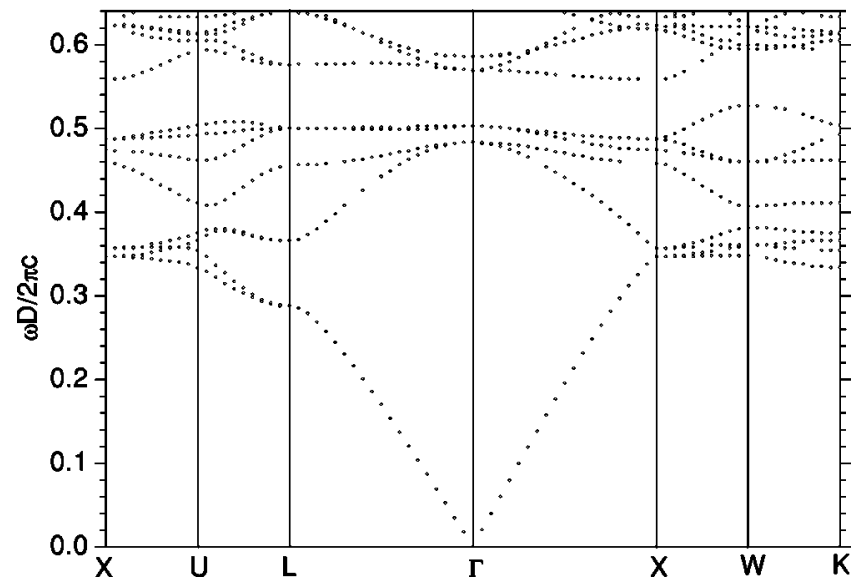

FIG. 2. Photonic band structure of an inverted opal composed of close-packed FCC lattice of touching air spheres in gallium arsenide. Other parameters are given in the text.

ond one is at $\tilde{\omega} \approx 0.484-0.498$ between fifth and sixth bands, and the third one is at $\tilde{\omega} \approx 0.503-0.570$ between the eighth and ninth bands. The third stop overlaps with the CPBG of the structure. The band structure of an inverted FCC opal photonic crystal in GaAs matrix is similar to that in silicon matrix [see Figs. 8(a) of Ref. [8]].
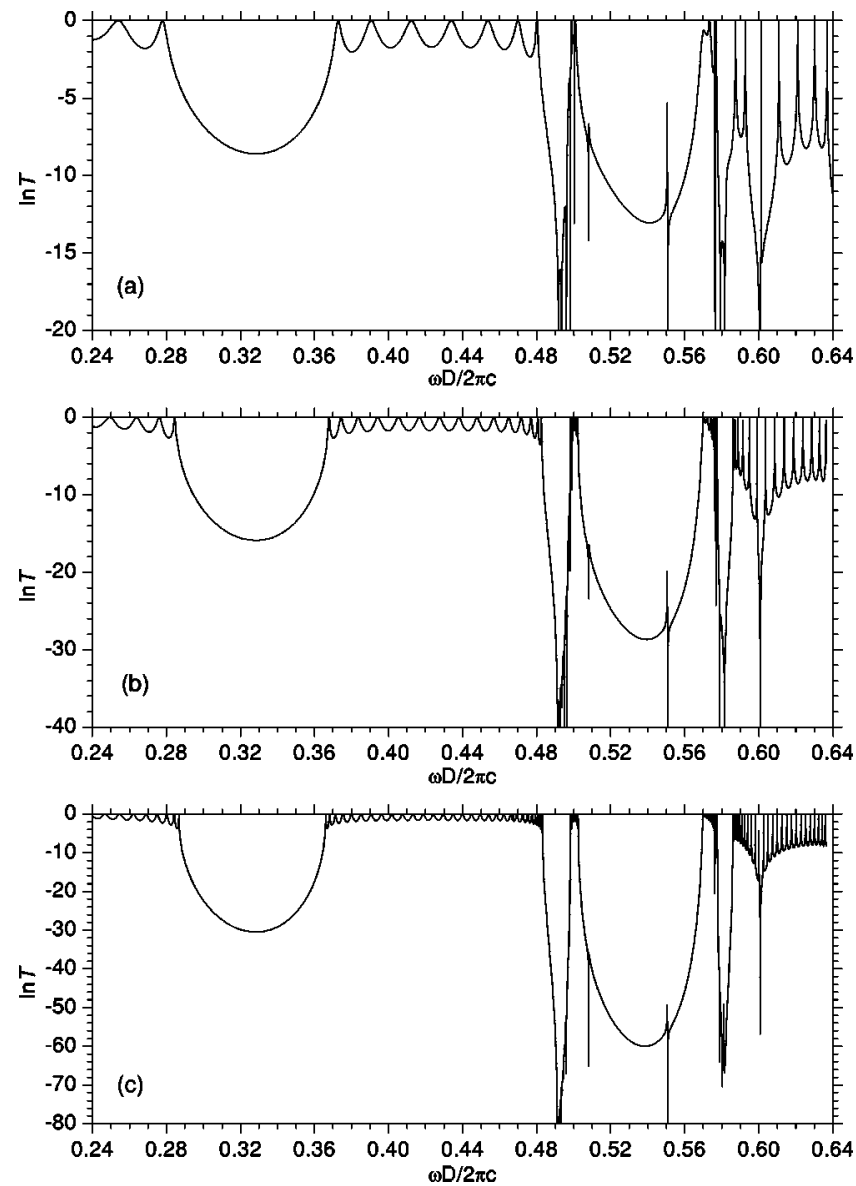

FIG. 3. Transmittance curves of light incident normally on slabs of the FCC PBG material consisting of 8 (a), 16 (b), and 32 (c) FCC (111) planes of touching air spheres in gallium arsenide. Other parameters are given in the text.
The dependence of the transmittance on thickness of the FCC inverted opal slabs is shown in Figs. 3(a), 3(b), and 3(c), where we plotted the transmission spectra for three slabs with 8, 16, and 32 (111) layers of air spheres, respectively. Transmittance curves display pronounced dips exactly within the three frequency regions of gaps along $\Gamma L$ symmetry mentioned above. For example, the dips in the transmittance for the slab consisting of 32 layers of air spheres are at $\tilde{\omega} \approx 0.287-0.366, \tilde{\omega} \approx 0.483-0.498$, and $\tilde{\omega} \approx 0.502-0.570$, which are in good agreement with the results deduced from the band structure calculation. Extremely small transmittance in these frequencies is observed because EM wave propagation is prohibited as a consequence of photonic band gap effect. The dips are also seen to deepen as the number of layers increases.

Two singularities in the transmittance curve are also seen within the third stop gap in Fig. 2. Although independent of the number of layers of spheres and the stacking sequences as will be seen in later discussions, the position of the singularities is found to change when we increase the value of the parameter $d_{s}$, where $d_{s}$ is the distance of the first layer of air spheres from the surface of the host medium (results not shown here) [33]. Although the underlying physical picture is not quite clear yet, the results indicate that the singularities could be related to the heterostructure effect at the interfaces between the homogeneous substrate chips and macroporous host medium.

In addition, we notice a narrow dip in the transmittance curves above the third stop gap, which is located at $\tilde{\omega}$ $\approx 0.577-0.586$ for the slab having 32 layers of spheres. Basically, this nonpropagating regime of frequency is not a result of photonic gap effect but stems from decoupling of the normally incident light to a nondegenerate band along $\Gamma L$ symmetry line, an effect that has been observed experimentally in 2D photonic crystals [34] and predicted theoretically in 3D photonic crystals with FCC geometry [31]. Here in order to display more clearly the decoupling effect in the FCC inverted opal, we have enlarged the transmission spectrum and the corresponding band structure in these frequencies and show the comparison in Fig. 4. As is seen in the figure, only the 11th (nondegenerate) band exists over this low-transmittance region, so the incident light is totally reflected due to the absence of coupling with the macroporous medium. Although an initial increase in the number of layers of spheres can lead to an increase in its depth (see Fig. 3), this narrow dip will saturate and cannot be further deepened when the number of layers of air spheres exceeds $N \geqslant 30$.

As the frequency goes above this symmetry-forbidden decoupling frequency regime, pass band region with strong oscillations in the transmittance is observed for all slabs under normal incidence. These peaks with high transmittance are so narrow that they may be difficult to observe in experiments if the spectral bandpass is not fine enough. As seen from Figs. 3(a), 3(b), and 3(c), the number of these states is determined by the number of planes of air spheres in the slab. These peaks are due to the excitation of the leaky modes that are confined to the interior of the slabs by the incident light field (see, e.g., Ref. [35]). It is noted that the 

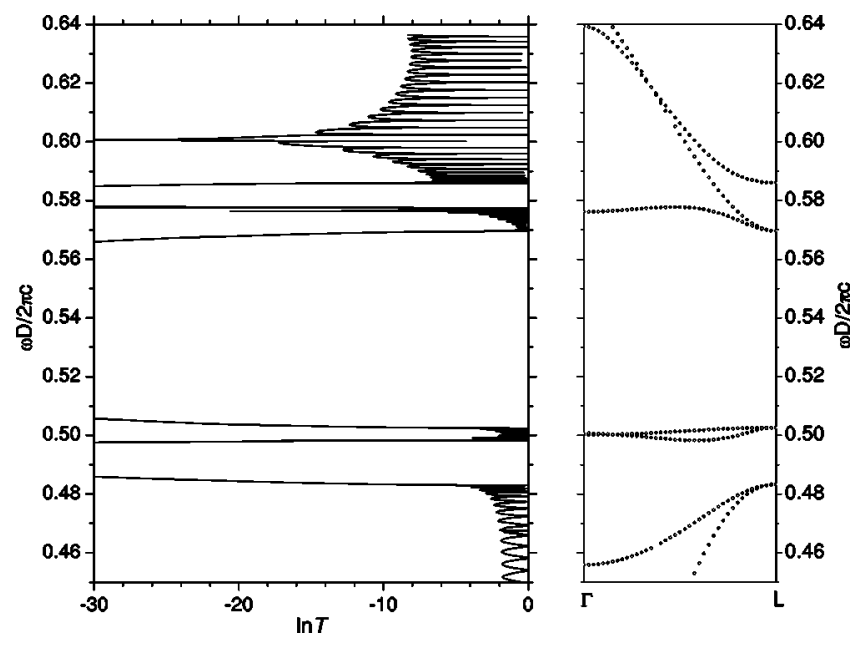

FIG. 4. Comparison of transmittance (left panel) of light normally incident on the slab of the inverted opal FCC PBG structure consisting of 16 (111) planes of touching air spheres [Fig. 3(b)] and the band structure (right panel) of the inverted opal FCC photonic crystal along $\Gamma L$ symmetry axis (Fig. 2). Other parameters are given in the text. The nonoptically active characteristics of the 11th band along $\Gamma L$ symmetry axis are exhibited clearly as a nontransmitting region in the transmission spectrum.

level distribution of these states depends on the local stacking configurations as well as the number of layers of air spheres in the slab (see later discussions).

The above absence of coupling of externally incident radiation with the nonoptically active band above the third gap can be removed by either moving away from the $\Gamma L$ symmetry line or by changing the symmetry via adopting $\ldots A B A B \ldots$ stacking order along $z$ axis, or even by breaking the periodicity with the introduction of stacking disorder. As the first evidence, we calculated the transmission spectra of an inverted FCC opal slab consisting of 16 layers of air spheres under off-normal incidence. In Fig. 5 we present the results for four different angles of incidence $\theta$ $=0,15^{\circ}, 25^{\circ}$, and $35^{\circ}$. The black lines are obtained for the $s$-polarized incident light where the electric field component of the incident light is perpendicular to the plane of incidence; the gray lines are obtained for the $p$-polarized incident light where the electric field component of the incident light lies in the plane of incidence. As expected, the nonoptically active mode becomes coupled to the incident radiation when the direction of incidence moves away from $L$ point, which leads to high transmittance in this region. This operation for coupling holds for both $s$ and $p$ polarizations. We also found that the first and third gaps shift to high frequencies, whereas the second gap shift to lower frequencies, and they all become narrower as the angle of incidence is increased.

\section{B. Inverted opal with HCP structure}

The HCP structure has a periodic stacking order with repeating $A B$ growth sequences. Band structure calculation using plane wave expansion has shown that the inverted HCP opal photonic crystal structure also possess a CPBG [8], provided that the refractive index of the host matrix is high enough as in the FCC case. We evaluated the photonic band structure and transmission spectrum along [0001] axis for an inverted HCP opal structure in GaAs matrix. The results are presented in Fig. 6. In the band structure shown, Fig. 6(a), we observed that the HCP structure possesses a CPBG between the 16th and 17th bands, which centers at $\tilde{\omega}_{0}=0.546$ with a relative size $\Delta \tilde{\omega} / \tilde{\omega}_{0}=3.8 \%$. The CPBG in HCP structure occurs at virtually the same frequency but has a smaller size as compared to the FCC case. A similar conclusion has been obtained by Busch and John [8] who studied the band structure of an inverted opal in silicon and showed that the CPBG of the HCP structure is somewhat smaller than that of the FCC structure. We also note that there are three stop gaps along $\Gamma A$ symmetry, which are located at $\tilde{\omega} \approx 0.288-0.365$, $\tilde{\omega} \approx 0.486-0.496$, and $\tilde{\omega} \approx 0.506-0.568$, respectively. Among the three gaps, the center and width of the first gap is the same as that of the FCC structure, while small variations in the band gap edges of the two higher gaps are seen when the periodic stacking unit is replaced with $A B$.

In Fig. 6(b), we plot the transmittance as a function of frequency for a HCP PBG material with 16 layers of touching air spheres in GaAs. The transmittance is calculated for light incident along the axis perpendicular to the hexagonally close-packing planes of air spheres which are referred to as (0001) plane in HCP structure. This direction corresponds to the $\Gamma A$ symmetry line in the band structure. In the figure, we observe three dips in the transmittance which are located at $\tilde{\omega} \approx 0.285-0.368, \tilde{\omega} \approx 0.484-0.496$, and $\tilde{\omega} \approx 0.506-0.568$, in good agreement with the three gaps found along $\Gamma A$ symmetry in band structure. We also notice that, instead of small transmittance in the FCC structure, a pass band with high transmittance above the third gap is observed in the inverted HCP photonic crystal since optically active band exists in this region for the HCP structure. In addition, the HCP structure also displays a strong oscillation feature in the transmittance at high frequencies but the distribution of energy levels of these modes are different from that seen in FCC geometry due to the different stacking configurations. Two singularities in the transmittance are also observed within the third gap and they are located almost at the same frequency even though the stacking unit here is changed from $A B C$ to $A B$.

\section{Inverted opal with DHCP structure}

A DHCP structure is another class of structure with periodic stacking order but with a larger stacking unit (four layers $A B C B)$. As a consequence, new band modes occur and the photonic band diagram becomes quite complex, particularly in the regions of higher-order bands. But similar to the FCC and HCP cases, the inverted opal DHCP structure exhibits a CPBG with a center frequency $\tilde{\omega}_{0}=0.543$, nearly the same position as in the two structures studied above. The DHCP structure, however, has a relative gap width $\Delta \tilde{\omega} / \tilde{\omega}_{0}$ $=6.0 \%$, which is substantially larger than the HCP structure, and slightly larger than the FCC structure. The value of the CPBG width obtained here by the photonic Korringa-KohnRostoker method is slightly larger than the value $(5.5 \%)$ obtained for the same system by plane wave expansion method [36]. We note that for systems consisting of spherical scat- 

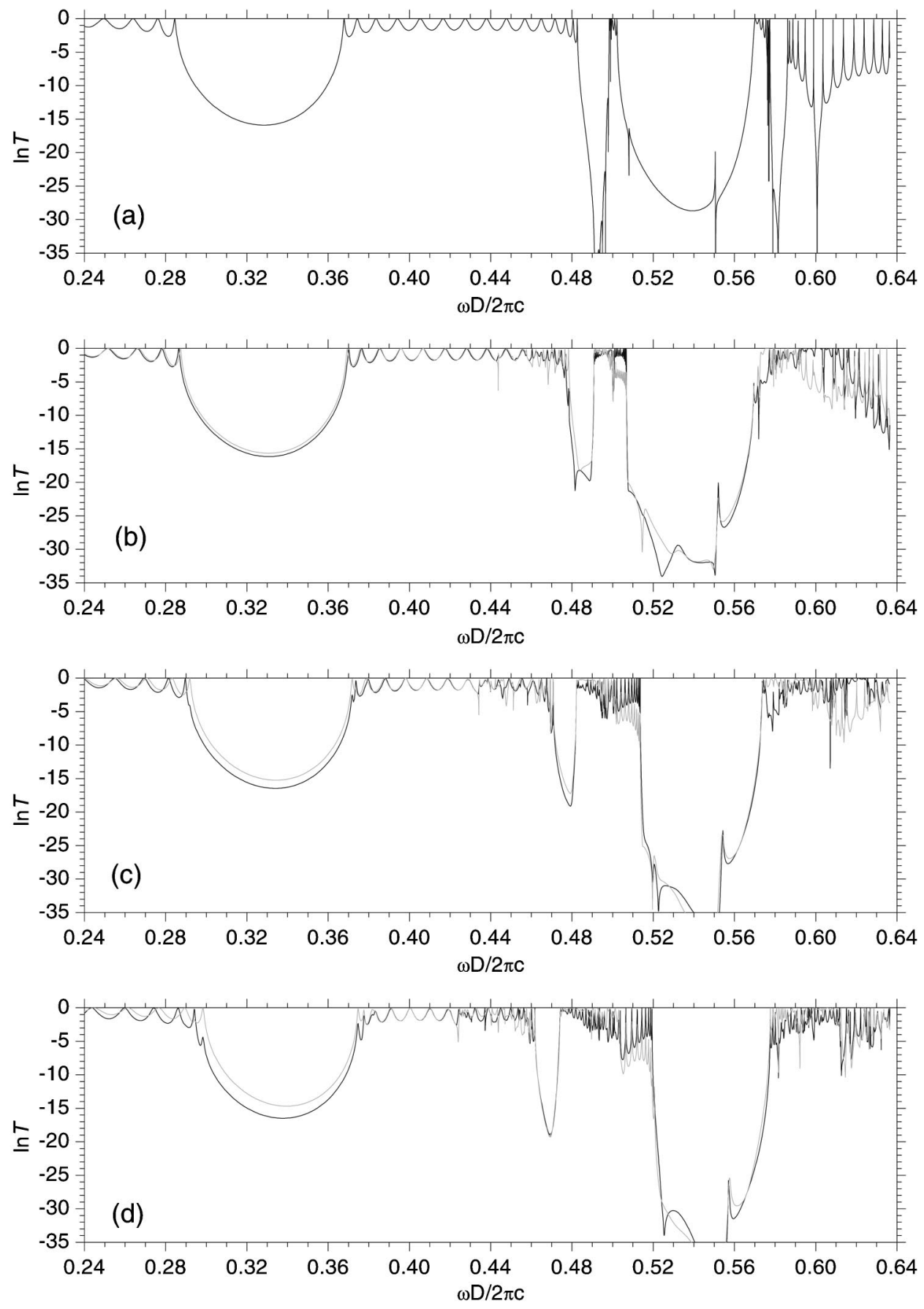

FIG. 5. Transmittance curves along the $L-W$ direction for the inverted opal FCC slab consisting of 16 (111) planes of touching air spheres in gallium arsenide. The black (gray) lines refer to $s$ (p) polarization of the incident light. (a) $L$ point, (b) $L+15^{\circ},(\mathrm{c}) L+25^{\circ}$, (d) $L$ $+35^{\circ}$. Other parameters are given in the text.

tering elements, the multiple scattering formalism usually gives more converged results than the plane wave expansion method does.

We have calculated the transmission of a DHCP photonic slab. Shown in Fig. 7(a) is the transmission spectrum along [0001] stacking direction of the DHCP structure having 16 layers of touching air spheres in GaAs. It is seen that, about the regions of frequency where pronounced dips are observed for FCC and HCP slabs, the transmission curve of the DHCP structure also shows three main pronounced dips.
These nontransmitting regions of frequency are the corresponding influences of the three stop gaps found in the band structure along $\Gamma A$ axis for the DHCP structure, which is displayed in Fig. 7(b) for comparison. In addition to these main gaps, the DHCP structure also develops two small gaps between the second and third main stop gap and these two small gaps are separated by two nondegenerate bands. Since for the region where there are only such nondegenerate band modes, the normally incident radiation will couple very weakly with the material, leading to extremely small trans- 

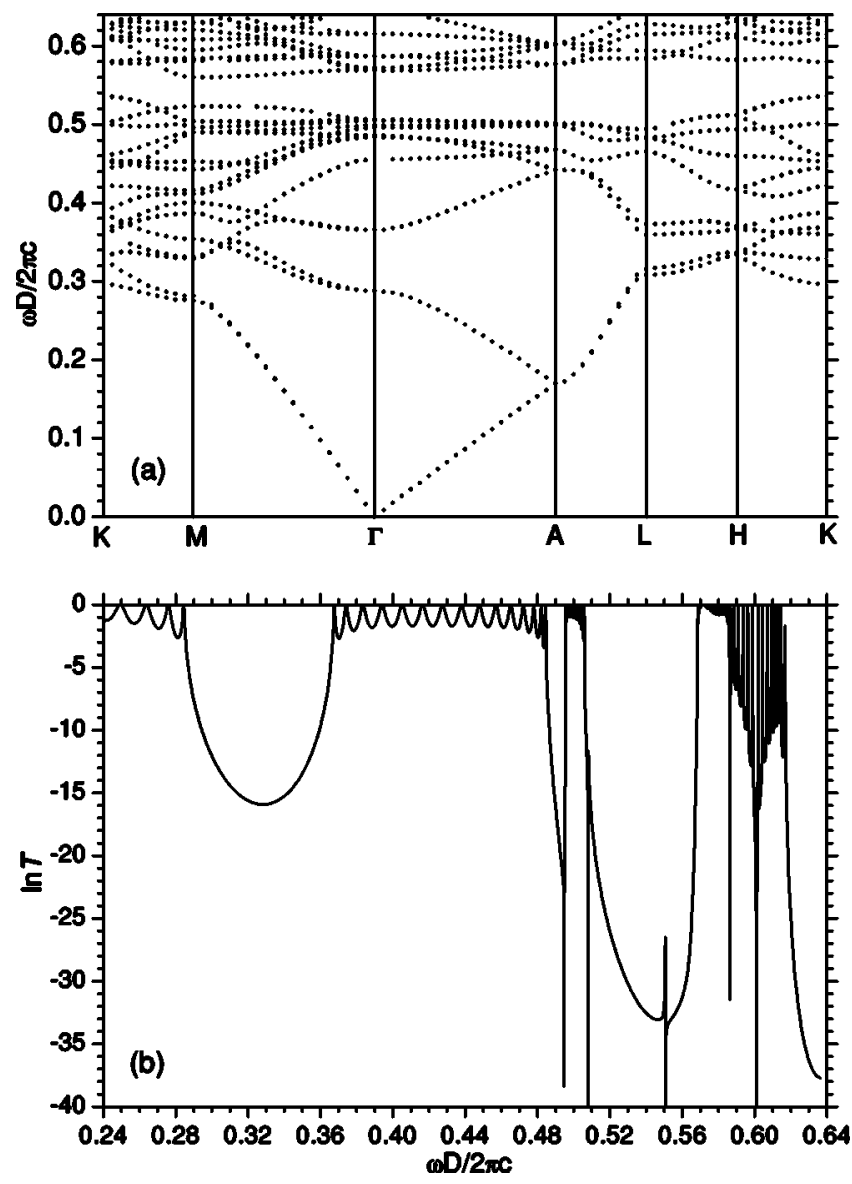

FIG. 6. (a) Photonic band structure of an inverted opal composed of close-packed HCP lattice of touching air spheres in gallium arsenide. (b) Transmittance of light normally incident on a slab of the HCP PBG material consisting of 16 (0001) layers of touching air spheres. Other parameters are given in the text.

mittance. This leads to a continuously vanishing transmittance in these regions of frequency, as is shown in the transmission spectrum in Fig. 7(a). Starting from the blue edge of the third stop gap $\tilde{\omega} \approx 0.571$, a nondegenerate band extends to $\tilde{\omega} \approx 0.580$. Over this spectral region, there are no other band modes except for two degenerate bands located from $\tilde{\omega} \approx 0.571$ to $\tilde{\omega} \approx 0.572$. This explains the nontransmitting feature immediately above the third stop gap in the transmittance curve, which is also a resultant of the decoupling of the external EM wave with the structure in this frequency region. Remember that the HCP structure is perfectly transmitting in the neighborhood above the blue edge of the third gap. Thus, in this regard, the DHCP is similar to the FCC case as we observed a low-transmittance region above the third gap for both structures due to the same reason. We may understand that a large fraction of $A B C$ stacking unit in the slab could favor the opening of a dip in transmission spectrum in this region.

\section{Inverted opal with random stacking disorder}

Although it preserves periodicity in the 2D hexagonal close packing plane, a structure composed of randomly
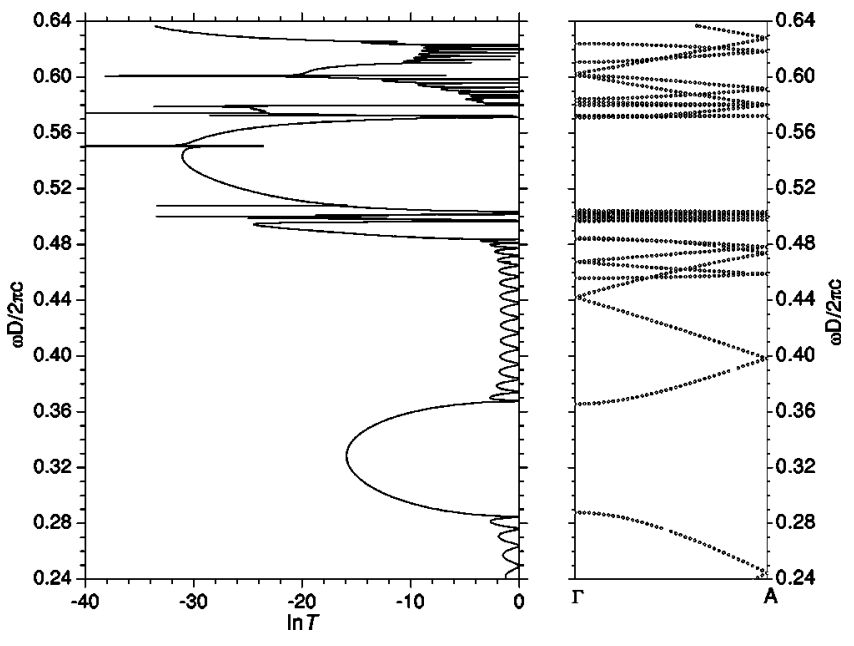

FIG. 7. Comparison of transmittance (left panel) of light normally incident on a slab of DHCP PBG structure consisting of 16 (0001) layers of touching air spheres and the band structure (right panel) of an inverted opal composed of close-packed DHCP lattice of touching air spheres in gallium arsenide along [0001] symmetry axis. Other parameters are given in the text.

stacked close-packed layers of air spheres is not a crystal, due to the loss of periodicity in another crystallographic direction (the growth direction). For the inverted opal structures with a random stacking sequences, the band structure is not well defined and it is not either practical to perform photonic band structure calculations for huge supercells due to computer time constraints. However, the transmittance through a slab of finite thickness is well defined, and so we calculate the transmittance and then average it over different stacking configurations to study the disorder influence on the transmission property of the structure. The disorder in our systems is introduced by randomizing the stacking sequence: $B$ and $C$ having equal occupancy probabilities. A discussion of small portion of and periodic stacking disorders in the inverted opal FCC structure can be found in Ref. [27].

We studied the transmittance of randomly stacked inverted opal slabs with three different numbers of layers of air spheres $N=16,32$, and 64 . In order to obtain the mean photonic properties of the stacking sequence disordered systems with a finite thickness, we averaged the logarithmic of the transmittance $(\ln T)$ over 100 stacking configurations. We have found that a further increase in the number of random realizations does not lead to distinguishable difference in $\langle\ln T\rangle$ from the case of 100 configurations.

In Fig. 8(a), we first display the result of one individual realization of the slab with random sequences of 64 layers of touching air spheres in GaAs. We observed that the transmission spectrum of the disordered structure also exhibits three pronounced dips in the regions of frequency, about the gaps of periodic structures. The occurrence of these dips in transmittance is due to the effect of strong light localization in these frequencies. The width and position of the first gap deduced from the first dip in transmission curve remain the same, while small variations in the edges of the two gaps at higher frequencies are observed as compared to the gaps of the periodic systems. This shows that the first gap of the 

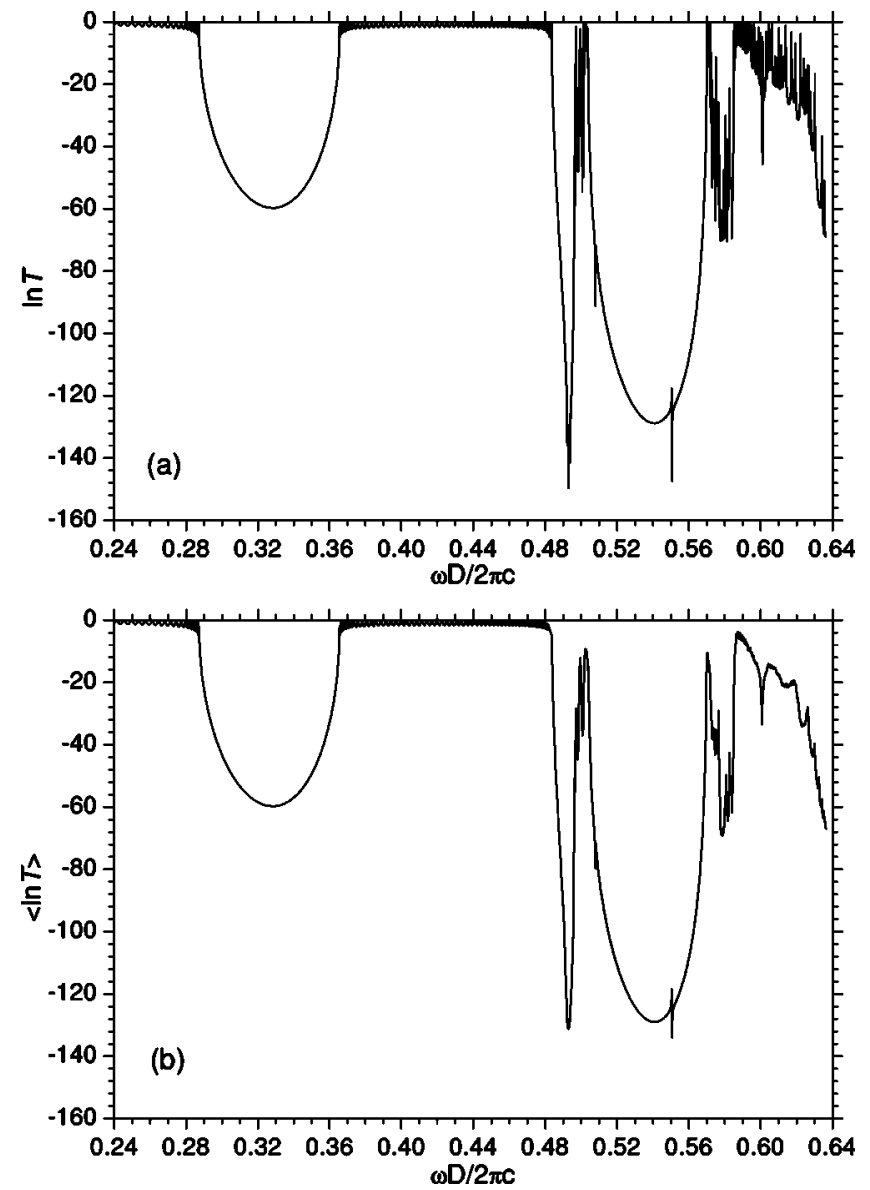

FIG. 8. Individual (a) and ensemble-averaged (b) logarithmic transmittance $\langle\ln T\rangle$ of light normally incident on a slab of inverted opal having 64 layers of hexagonally touching air spheres with random stacking sequences in gallium arsenide. Other parameters are given in the text.

inverted opal photonic crystal is almost independent of the stacking sequence in the opal template; the two gaps at higher energy can still survive although small variation in their edges occurs as a consequence of defect states created near the edges of the gaps of the periodic systems.

On the other hand, with the introduction of random stacking sequence, wild oscillations in the transmittance develop in the region of frequency around the red and blue edges of the third gap of the periodic systems. This phenomenon is anticipated as we have shown above that the transmission property changes dramatically in these regions even for periodic systems with different stacking units. Thus, for example, any small deviation in stacking sequences from the ordered $A B C A B C \ldots$ stacking configuration should introduce propagation band modes in the region above the third stop gap where only nonoptically active nondegenerate band modes exist for the FCC structure. At higher frequencies that start at $\tilde{\omega} \approx 0.58$, the strong oscillating feature observed in systems with ordered stacking sequences preserves for the random system. It is found that the energy level of these leaky modes associated with narrow high transmittance is also sensitive to the local stacking configuration.

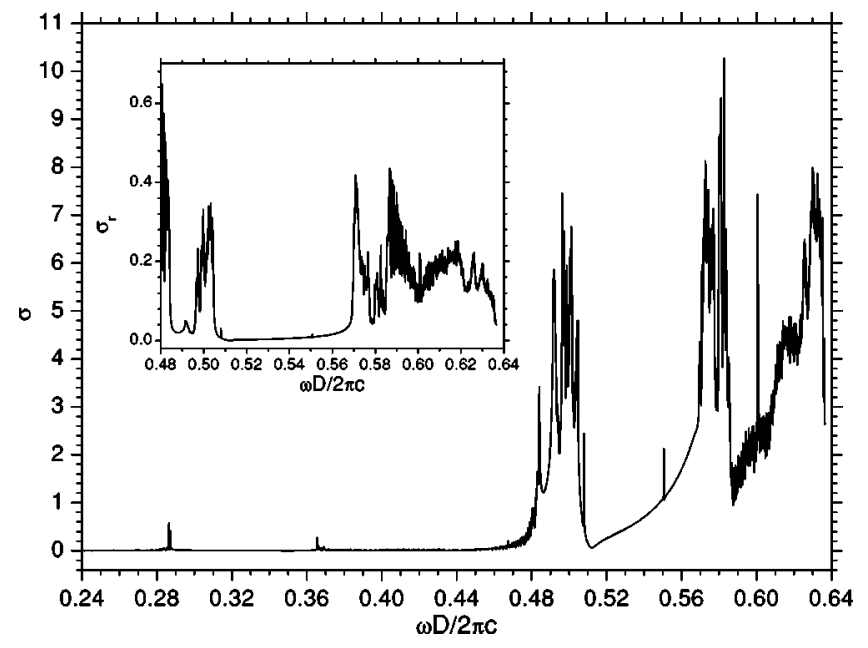

FIG. 9. Standard error of $\langle\ln T\rangle$ as a function of frequency for slabs of inverted opal having 64 layers of hexagonally touching air spheres randomly stacked in gallium arsenide. Other parameters are given in the text. Shown in the inset is the relative standard error of $\langle\ln T\rangle$ vs frequency in the region from $\tilde{\omega}=0.48$ to $\tilde{\omega}=0.64$.

Plotted in Fig. 8(b) is the ensemble-averaged logarithmic transmittance $\langle\ln T\rangle$ as a function of frequency for inverted opal slabs consisting of randomly distributed sequences of 64 layers of air spheres in GaAs. The transmission spectrum below $\tilde{\omega} \approx 0.48$ is almost the same as that of structures with periodic stacking unit. The third stop gap at high frequency, which overlaps with the CPBG in periodic systems, survives stacking disorder although the transmittance at its red and blue edges is suppressed due to light localization effect. This contrasts with the disorder in position of air spheres that was found to destroy the high-frequency absolute gap $[26,36]$. We thus conclude that for inverted opal photonic crystals to function, it is important to have a good control of the size uniformity of the spheres, and hopefully the monodispersity

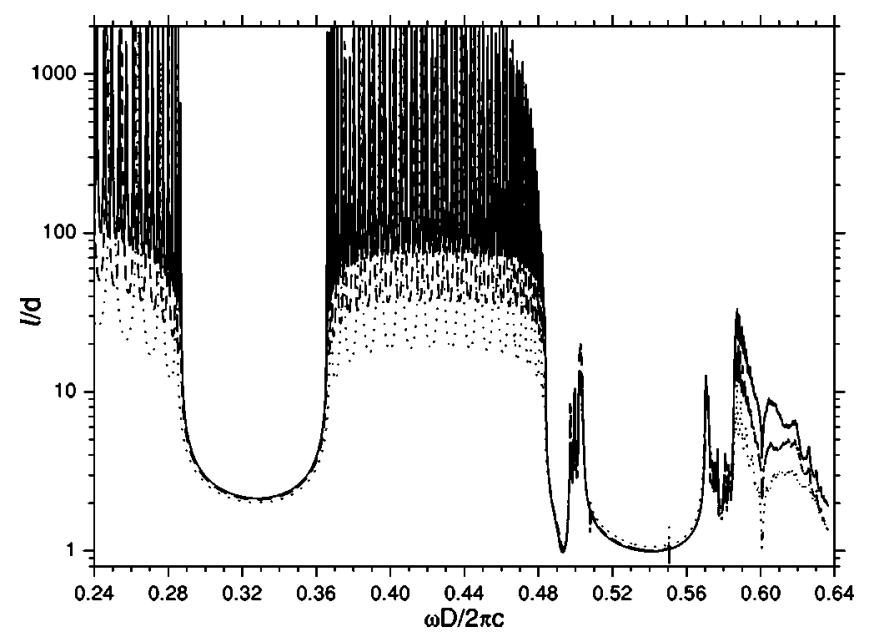

FIG. 10. $l / d$ as functions of frequency for inverted opals with random stacking sequences. The dotted, dashed, and solid lines are for slabs of inverted opal having 16, 32, and 64 layers of hexagonally touching air spheres randomly stacked in gallium arsenide, respectively. Other parameters are given in the text. 
can minimize the positional disorder in opal templates.

For the disordered structures, another remarkable change is the development of a diminishing transmittance spectral region over wide frequencies above the third gap. The narrow dip immediately above the third gap can be understood as follows. We remember that over this region, both FCC and DHCP structures develop nontransmitting spectra, whereas total transmittance is observed for a HCP structure. This suggests that any occurrence of $A B C$ in the stacking sequence in random system favors, to some extent, the appearance of a dip at these frequencies, although the specific positions may change slightly for different configurations. On the other hand, the physics underlying the strongly reduced transmittance at higher frequencies starting at $\tilde{\omega}=0.59$ is due to weak light localization, and can be examined more quantitatively by plotting the localization length for different thickness of the slabs in later sections.

We also calculated the standard error $\sigma$ that characterizes the fluctuation of individual $\ln T$ from the mean value $\langle\ln T\rangle$. The $\sigma$ is defined as $\sigma=\Sigma\left|\delta_{i}\right| / N_{c}$, where $\delta_{i}=\ln T-\langle\ln T\rangle$ is the deviation of the individual measurement of $\ln T, N_{c}$ is the number of configurations, and the sum runs over all random stacking configurations. In Fig. 9, $\sigma$ is plotted as a function of frequency for inverted opal photonic slabs with 64 layers of touching air spheres in GaAs. We observed that below $\tilde{\omega} \approx 0.48$, the fluctuation in transmittance is very weak although a small error mainly at the edges of the first gap is observed. Consequently, the transmission property of the slab is not affected by the stacking disorder when the frequency is lower than $\tilde{\omega} \approx 0.48$. This means that the position and width of the lowest gap along stacking direction remain intact, regardless of the stacking sequence. In the frequency region where the third dip survives under planar stacking disorder, the standard deviation of $\langle\ln T\rangle$ is also small in this region. This can be seen more clearly in the inset of Fig. 9, where we display the relative standard error $\sigma_{r}\left(\sigma_{r}\right.$ $=\sigma /\langle\ln T\rangle)$ at these frequencies, which measures the relative fluctuation of $\langle\ln T\rangle$. These results show that defect states introduced by staking disorder are more located near the edges of the gaps, which leads to the preservation of the gaps along stacking direction even under the influence of random stacking sequences. Nevertheless, in the presence of strong disorder in stacking sequence, large fluctuations in transmittance, which are generally larger than $12 \%$, are observed near the blue edges of the two gaps at higher frequency and in the frequency region beyond $\tilde{\omega} \approx 0.585$. This contrasts to the small relative standard error in the region inside the gaps. We note that for periodic systems such as FCC and DHCP structures, decoupling phenomenon usually happens in the pass bands near the blue edges of the two higher stop gaps. This suggests that the inverted opal may become coupled to the incident EM wave via the creation of optically active band modes in these regions as a consequence of disorder perturbation in the stacking sequences. The highly transmitting leaky modes observed in the region above $\tilde{\omega} \approx 0.585$ are sensitive to the local stacking configurations, so different realization of the stacking sequences will result in strong fluctuation in transmittance in the region.
In order to describe the transport property of EM waves in a random dielectric medium, a localization scaling length $l$ is usually introduced in numerical calculations with $l / d=$ $-2 N /\langle\ln T\rangle$, where $d$ is the distance between neighboring planes of air spheres (see, e.g., Ref. [37]). In Fig. 10, we show the results for inverted opal photonic structures in the matrix of GaAs with random stacking sequences containing three different number of layers of air spheres $N=16,32$, and 64 , where $l / d$ is plotted as a function of frequency. The localization length tends to converge in the regions of frequency, about the gaps of periodic systems, when the number of layers of air spheres is increased from 32 to 64. For these spectrum regions, where the uncertainty of $\langle\ln T\rangle$ is very small (see Fig. 9), $l / d$ can be well approximated as the Anderson localization length. This suggests that all three photonic gaps remain well defined in inverted opal photonic crystal even in the presence of random stacking sequences.

As pointed by Asatryan et al. [37] in the study of disorder in $2 \mathrm{D}$ photonic crystals with a finite thickness, $l / d$ incorporates contributions from both Anderson localization and Fabry-Perot interference effect. In Fig. 10, the localization effect due to light interference among layers of air spheres in the inverted opal is observed in the region of frequencies, about the pass bands below the second gap of the periodic systems, where $l / d$ shows oscillatory behavior. This property is consistent with the fact that the localization length in these regions is usually larger than the slab thickness, at least for slabs with 32 and 64 layers of air spheres. For the regions at $\tilde{\omega} \approx 0.495-0.505$ and $\tilde{\omega} \approx 0.572-0.585$, a reduction of localization length is also observed but generally is accompanied with large fluctuations in the mean value (see Fig. 9), which indicates an effect of weak localization in these frequency regions. In EM wave propagation, the effective perturbation due to structural disorder, as manifested in the variation in the positional dependence of dielectric constant, is weighted by a factor of $\omega^{2}$. Thus localization effect will become more noticeable as frequency goes up. This may explain the reduced localization length in the frequency region ( $\tilde{\omega}$ $>0.585$ ) where a localization length on the order of a few layer thickness is observed. However, $l / d$ does not converge for the inverted opal photonic crystals considered, so light localization is still weak in this region even for the slabs with 64 layers of air spheres.

\section{CONCLUSIONS}

Using EM wave multiple scattering technique, we have studied the effect of different stacking sequences on the optical properties of inverted opal photonic crystals composed of close-packed air spheres embedded in high dielectric matrix of GaAs.

We show that all the periodic structures with FCC, HCP, and DHCP lattices possess complete photonic band gap with slight variations in the gap edges. Along the symmetry axis, which corresponds to the direction perpendicular to the planes of hexagonal close-packed air spheres, all the periodic photonic crystals exhibit three stop gaps, with the first one being identical, whereas a slight variation in the gap edges of the second and third stop gaps is observed. Excellent agree- 
ment between the band structure and the transmission spectrum along stacking direction are obtained for all these periodic systems.

We also observed nontransmitting regions of spectrum for the periodic structures for light normally incident on the planes of hexagonally close-packing air spheres. The phenomenon is well explained as a consequence of the existence of only nondegenerate modes without any other degenerate states in the region in the band structure. Since the nondegenerate modes are nonoptically active for the normally incident light due to symmetry reason, this leads to the nontransmitting property in the regions of pass band. It is further shown that coupling of the externally incident light to the material becomes possible by adopting off-axis incidence, by changing the symmetry along stacking direction, or by introducing disorder in the stacking sequences.

For inverted opals with a totally random stacking sequence, the calculated transmission spectrum also develops pronounced dips within the frequencies of the stop gaps of the periodic systems. We conclude that the stop gaps in inverted opal are fairly robust to stacking disorder. The lowest stop gap is basically untouched by stacking randomness. This is due to the fact that the effective perturbation is weaker in lower frequencies. Large fluctuations are observed near the edges of the two high stop gaps as a consequence of the creation of defect states induced by stacking disorder.
The uncertainty of transmittance in the region, about the gaps of the periodic structure, is small because deep gap states are rarely introduced. This means that these systems can withstand a fair amount of stacking randomness so that we do not need to worry too much about the stacking disorder. However, light localization can be an issue for applications that utilize the pass band or if we need to couple light in or out of the crystals in frequencies near the absolute gap. For slabs with reasonable thickness, low-frequency pass bands such as those near the lowest directional gap show propagation behavior even when the stacks are completely disordered in stacking sequences. However, for pass bands near the absolute gap, disorder in stacking sequences can make the localization length fairly small, e.g., a few layers.

\section{ACKNOWLEDGMENTS}

This work was supported in part by the key research project in "Climbing Program" by the National Science and Technology Commission of China and RGC through HKUST6145/99P. Z.W. acknowledges partial financial support from the Natural Science Foundation of China (NSFC) under Grant Nos. 10174031 and 90101030. W.Z. also acknowledges partial support from NSFC under "Excellent Youth Foundation."
[1] E. Yablonovitch, Phys. Rev. Lett. 58, 2059 (1987).

[2] S. John, Phys. Rev. Lett. 58, 2486 (1987).

[3] J.D. Joannopoulos, P.R. Villeneuve, and Shanhui Fan, Nature (London) 386, 143 (1997).

[4] Yu.A. Vlasov, V.N. Astratov, O.Z. Karimov, A.A. Kaplyanskii, V.N. Bogomolov, and A.V. Prokofiev, Phys. Rev. B 55, R13357 (1997).

[5] R. Mayoral, J. Requena, S.J. Moya, C. López, A. Cintas, H. Míguez, F. Meseguer, L. Vázquez, M. Holgado, and A. Blanco, Adv. Mater. 9, 257 (1997).

[6] S.H. Park and Y.N. Xia, Adv. Mater. 10, 1045 (1998).

[7] H.S. Sözüer, J.W. Haus, and R. Inguva, Phys. Rev. B 45, 13 962 (1992).

[8] K. Busch and S. John, Phys. Rev. E 58, 3896 (1998).

[9] A. Moroz and C. Sommers, J. Phys.: Condens. Matter 11, 997 (1999).

[10] A. Velev, T.A. Jede, R.F. Lobo, and A.M. Lenhoff, Nature (London) 389, 447 (1997).

[11] A. Imhof and D.J. Pine, Nature (London) 389, 948 (1997).

[12] A.A. Zakhidov, R.H. Baughman, Z. Iqbal, C. Cui, I. Khairulin, S.O. Dantas, J. Marti, and V.G. Ralchenko, Science 282, 897 (1998).

[13] Yu.A. Vlasov, N. Yao, and D.J. Norris, Adv. Mater. 11, 165 (1999).

[14] P.V. Braun and P. Wiltzius, Nature (London) 402, 603 (1999).

[15] B.T. Holland, C.F. Blandford, and A. Stein, Science 281, 538 (1998).

[16] J.E.G.J. Wijnhoven and W.L. Vos, Science 281, 802 (1998).

[17] G. Subramania, K. Constant, R. Biswas, M.M. Sigalas, and
K.-M. Ho, Appl. Phys. Lett. 74, 3933 (1999).

[18] M. Müller, R. Zentel, T. Maka, S.G. Romanov, and C.M. Sotomayor Torres, Adv. Mater. 12, 1499 (2000).

[19] A. Blanco, E. Chomski, S. Grabtchak, M. Lbisate, S. John, S.W. Leonard, C. Lopez, F. Meseguer, H. Miguez, J.P. Mondia, G.A. Ozin, O. Toader, and H.M. van Driel, Nature (London) 405, 437 (2000).

[20] Yu.A. Vlasov, X.Z. Bo, J.C. Sturm, and D.J. Norris, Nature (London) 414, 289 (2001).

[21] Yu.A. Vlasov, V.N. Astratov, A.V. Baryshev, A.A. Kaplyanskii, O.Z. Karimov, and M.F. Limonov, Phys. Rev. E 61, 5784 (2000).

[22] L.V. Woodcock, Nature (London) 385, 141 (1997); 388, 236 (1997).

[23] A. van Blaaderen and P. Wiltzius, Science 270, 1177 (1995).

[24] H. Versmold, Phys. Rev. Lett. 75, 763 (1995).

[25] N.A. Verhaegh, J.S. van Duijneveldt, A. van Blaaderen, and H.N.W. Lekkeerkerker, J. Chem. Phys. 102, 1416 (1995).

[26] Z.-Y. Li and Z.-Q. Zhang, Phys. Rev. B 62, 1516 (2000).

[27] V. Yannopapas, N. Stefanou, and A. Modinos, Phys. Rev. Lett. 86, 4811 (2001).

[28] K. Ohtaka, J. Phys. C 13, 667 (1980).

[29] X.D. Wang, X.G. Zhang, Q.L. Yu, and B.N. Harmon, Phys. Rev. B 47, 4161 (1993).

[30] C.T. Chan, W.Y. Zhang, Z.L. Wang, X.Y. Lei, D.G. Zheng, W.Y. Tam, and P. Sheng, Physica B 279, 150 (2000).

[31] N. Stefanou, V. Karathanos, and A. Modinos, J. Phys.: Condens. Matter 4, 7389 (1992).

[32] N. Stefanou, V. Yannopapas, and A. Modinos, Comput. Phys. Commun. 113, 49 (1998). 
[33] For a system with $d_{s}>D / 2$, the photonic crystal can be viewed as a macroporous medium of GaAs sandwiched by two identical homogeneous slabs of GaAs with a thickness of $d_{s}$ $-D / 2$.

[34] W.M. Robertson, G. Arjavalingam, R.D. Meade, K.D. Brommer, A.M. Rappe, and J.D. Joannopoulos, Phys. Rev. Lett. 68, 2023 (1992).
[35] V. Karathanos, J. Mod. Opt. 45, 1751 (1998).

[36] R. Biswas, M.M. Sigalas, G. Subramania, C.M. Soukoulis, and K.-M. Ho, Phys. Rev. B 61, 4549 (2000).

[37] A.A. Asatryan, P.A. Robinson, L.C. Botten, R.C. McPhedran, N.A. Nicorovici, and C. Martijn de Sterke, Phys. Rev. E 60, 6118 (1999). 